\title{
Finite element assessment of the effects of seismic loading rate on soil liquefaction
}

\author{
Radu Popescu
}

\begin{abstract}
The influence of frequency content of seismic excitation on the extent and pattern of pore-water pressure build-up in saturated soil deposits is addressed. Seismic acceleration time histories are generated as uniformly modulated nonstationary stochastic processes, in accordance with prescribed response spectra and prescribed modulating functions. Based on numerical examples, it is shown that the interplay between the frequency content of seismic excitation and the dynamic characteristics of the soil system and their evolution during and after the earthquake have important implications on the dynamic response.
\end{abstract}

Key words: seismic motion, frequency content, finite elements, soil dynamics.

Résumé : L'influence de la fréquence de l'excitation séismique sur la magnitude et la configuration de la pression d'eau interstitielle dans les dépôts de sol saturé a été étudiée. Des accélérations séismiques sont simulées en tant que processus stochastiques uniformément modulés, selon des spectres de réponse et des fonctions de modulation prescrits. On a démontré que l'interaction entre la fréquence de l'excitation séismique et les caractéristiques dynamiques du sol ont des implications importantes sur la réponse dynamique.

Mots clés : mouvement séismique, fréquence, éléments finis, dynamique des sols.

\subsection{Introduction}

The characteristics of seismic ground motion at a specific location (e.g., frequency content, amplitude) are mainly governed by: (1) the distance from the seismic source, (2) local soil conditions, and (3) the magnitude of the event. As a consequence of wave propagation and loss of coherence, there is a certain spatial variation of seismic ground motion from one location to another. To this end, the earthquake ground motion can be described by a nonstationary stochastic vector process with evolutionary power, each scalar component of the vector process representing the motion at a certain spatial location (e.g., Deodatis 1996).

As dynamic systems, any soil deposit or soil-structure ensemble has their own characteristic frequency (or lower eigenfrequency value), which depends on soil properties (especially on shear modulus), geometry, and degree of saturation. This characteristic frequency may decrease during dynamic excitation, due to degradation of the effective shear moduli as a result of pore pressure build-up and (or) large shear strains. This phenomenon has been documented by Madabhushi and Schofield (1993). Based on a series of centrifuge experiments of tower-soil systems, they concluded that when the dominant frequency of the seismic excitation was lower than the natural frequency of their model, buildup of excess pore pressure and subsequent degradation of

R. Popescu. Faculty of Engineering \& Applied Science and C-CORE, Memorial University of Newfoundland, St. John's, NF A1B 3X5, Canada. (e-mail: radu@engr.mun.ca). soil stiffness was lowering the initial natural frequency of the tower-soil system, to a value close to the seismic input frequency.

Based on two examples of liquefaction potential assessment, it is shown in this paper that the interplay between the frequency content of seismic excitation and the dynamic characteristics of soil deposits or earth structures, and especially their evolution during and after the earthquake, has important implications on the dynamic behaviour. Nonlinear dynamic finite element analyses are carried out using a multiyield plasticity model implemented in the computer code DYNAFLOW (Prevost 1999). The calculations are conducted in terms of effective stress, using fully coupled solid fluid equations for the treatment of saturated porous media.

\subsection{Digital generation of seismic accelerations}

\subsection{Simulation algorithm}

Seismic design codes provide response spectra for various types of local site conditions. Acceleration, velocity or displacement time histories that are compatible with the recommended response spectra are used as input motion in dynamic computations. Such time histories are usually generated at several locations on the ground surface, as sample functions of a nonstationary, multivariate stochastic process. A procedure for digital generation of nonstationary stochastic processes is used here to generate seismic accelerations.

Sample functions, $f^{0}(t)$, of a uniformly modulated, nonstationary, multivariate stochastic process, $f(t)$, are generated using a spectral representation based algorithm. This algorithm, implemented in the computer package PRISM 
(Popescu et al. 2000), is a special case of the simulation algorithm for nonstationary vector processes proposed by Deodatis (1996). The resulting sample functions are compatible with prescribed response spectra, correlated according to a given coherence function, include the wave propagation effect, and have prescribed modulating functions for amplitude variation. The algorithm takes advantage of the great computational efficiency of the Fast Fourier Transform technique.

Each simulated time history is a scalar component of the nonstationary, multivariate sample function $f^{0}(t)$, and corresponds to a specific location on the ground surface. Each scalar component is expressed as

$$
f_{j}^{0}(t)=A_{j}(t) g_{j}^{0}(t) \quad j=1, \ldots, N
$$

where $N$ is the total number of points on the ground surface where correlated seismic motions are simulated; $A_{j}(t)$ are the modulating functions (envelopes); and $g_{j}^{0}(t)$ are sample functions of a stationary stochastic process.

The Jennings et al. (1968) model is selected for the modulating functions $A_{j}(t)$ that control the duration of the strong ground motion. The coherence functions, describing the correlation between the scalar components, follow the Abrahamson (1993) model, which can be used for a broad range of soil conditions. The effects of wave propagation are introduced by prescribing the locations on the ground surface where seismic motion is simulated and the velocity of seismic wave propagation.

The simulation starts by generating ergodic, stationary time histories, $g_{j}^{0}(t)$, using the spectral representation method. The stationary sample functions are then modulated with respect to prescribed envelope functions, as shown in eq. [1]. The resulting sample functions are compatible with prescribed coherence functions and include the effects of wave propagation, but they are not compatible with the prescribed (target) response spectra. Iterative upgrading of the power spectral density functions of each scalar component, $g_{j}^{0}(t)$, is performed next, until the resulting response spectra of the nonstationary time histories, $f_{j}^{0}(t)$, are within an acceptable tolerance of the targets. Less than 10 iterations are usually needed. For a detailed description of the generation algorithm the reader is referred to Deodatis (1996).

\subsection{Acceleration time histories used in this study}

To assess the effects of loading rate (frequency content of seismic excitation) on dynamic behavior, earthquake ground motions corresponding to different local soil conditions are simulated using four different prescribed response spectra shown in Fig. 1a. Specifically, the first three types of response spectra plotted in Fig. 1a correspond to the Uniform Building Code (UBC) (1994), with Type 1 for rock and stiff soils, Type 2 for deep cohesionless or stiff clay soils, and Type 3 for soft to medium stiff clays and sands. The response spectrum labeled as Type 4, with a range of maximum spectral values corresponding to frequencies that are lower than for Types 1, 2, and 3, is believed to be representative for locations close to the epicenter. The effectiveness of the simulation algorithm is illustrated in Fig. 2, where the prescribed (target) response spectra (continuous lines) are compared with the response spectra computed from the corresponding simulated acceleration time histories (represented by dotted lines).

For demonstration purposes, the synthesized acceleration time histories presented in Figs. $1 b-1 e$ are used as base input motions in the numerical examples presented in Sections 4 and 5. Identical modulating functions, $A_{j}(t), j=1, \ldots, 4$, in eq. [1], are used for all of the acceleration time histories. They are generated at close locations on the ground, so there is no significant loss of coherence. The acceleration time histories presented in Fig. 1 have been scaled to maximum values of about $0.15 \mathrm{~g}$, to represent earthquakes delivering the same amounts of energy (or having similar intensities). This is illustrated in Fig. $3 a$, by the evolution of Arias Intensity values computed for the four time histories. Arias Intensity index is a measure of the total energy delivered per unit mass during an earthquake, and can be expressed as (Arias 1970)

$$
I=\frac{\pi}{2 g} \int_{0}^{T} a^{2}(t) \mathrm{d} t
$$

where $T$ is the total duration of the earthquake, and $a(t)$ is the ground acceleration at instant $t$. To estimate the amount of energy delivered up to any time instant, $t$, the values $I(t)$ shown in Fig. $3 a$ are computed as

$$
I(t)=\frac{\pi}{2 g} \int_{0}^{t} a^{2}(\tau) \mathrm{d} \tau
$$

Another measure of the seismic intensity of an acceleration time history is the number of representative cycles at 0.65 $a_{\max }$. The number of representative cycles from the beginning of the earthquake to any time instant, $t$, is computed for the four acceleration time histories presented in Fig. 1 using a method proposed by Seed et al. (1975). These numbers are represented in Fig. $3 b$. It results from Fig. $3 b$ that the four acceleration time histories used in this study are similar with respect to the numbers of representative cycles, at least up to time $t=8 \mathrm{~s}$. After $t=8 \mathrm{~s}$, the Type 4 acceleration time history has about $15 \%$ less representative cycles than the other three seismic accelerations.

\subsection{Mathematical model}

The four acceleration time histories presented in Fig. 1 are used as seismic input motion for dynamic analyses of a soil deposit and of an embankment dam. Nonlinear dynamic finite element analyses are performed using the code DYNAFLOW (Prevost 1999). DYNAFLOW is a finite element analysis program for the static and transient response of linear and nonlinear two- and three-dimensional systems. The solid and fluid coupled field equations are based on an extension of Biot's formulation (Biot 1962) in the nonlinear regime, and are applicable to multidimensional situations. A multiyield constitutive model is used for simulating the behaviour of soil materials. It is a kinematic hardening model based on a simple plasticity theory (Prevost 1985), and it is applicable to both cohesive and cohesionless soils. The yield function is described in the principal stress space by a set of nested conical yield surfaces. A non-associative plastic flow rule is used for the dilatational component of the plastic deformation. The model has been tailored (1) to retain the extreme versatility and accuracy of the simple multisurface $\mathrm{J}_{2}$ theory in describ- 
Fig. 1. Digital generation of response spectrum compatible seismic accelerations: $(a)$ types of prescribed response spectra; $(b)-(e)$ response spectrum compatible acceleration time histories used in this study. UBC, Uniform Building Code.
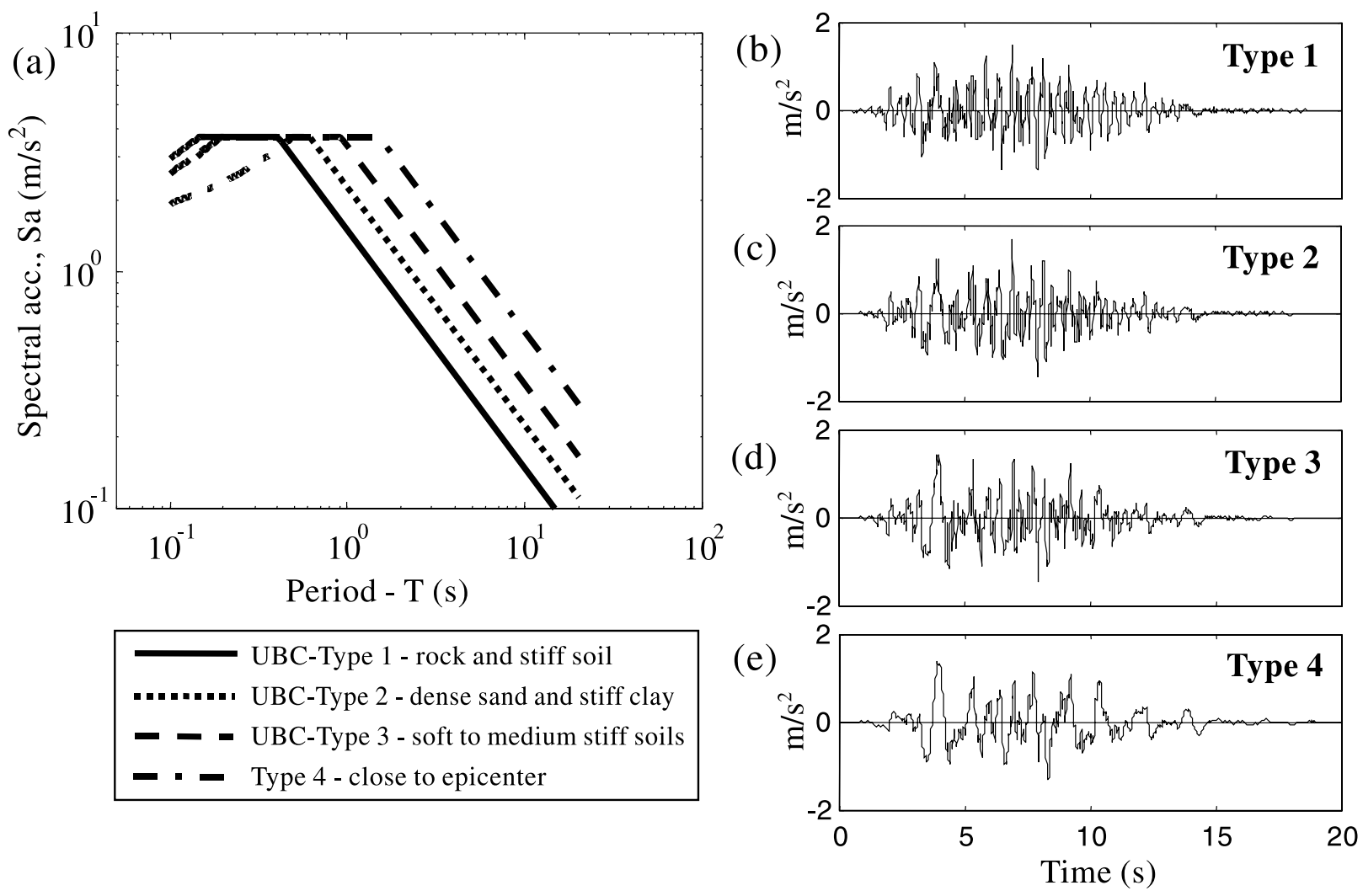

Fig. 2. Digital generation of response spectrum compatible seismic accelerations: comparison between prescribed and resulting acceleration response spectra.

(a)

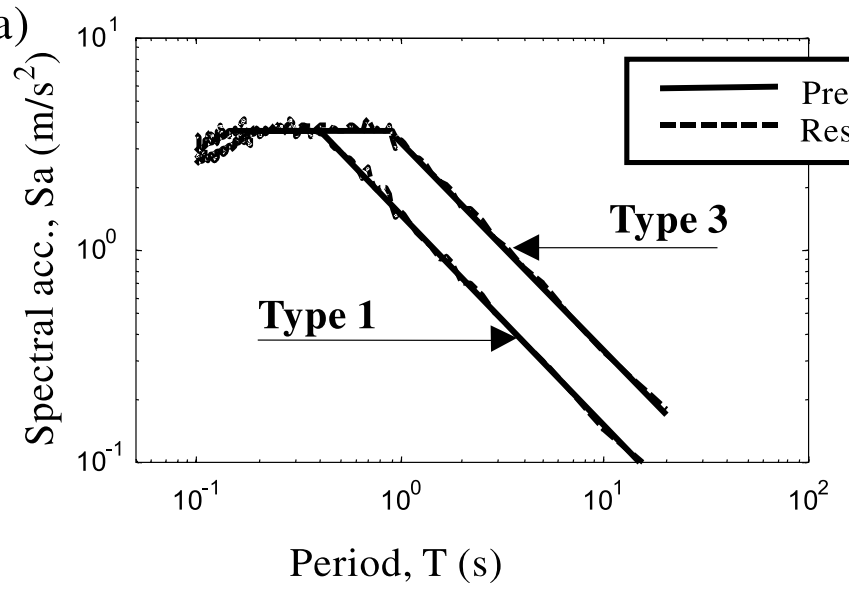

ing observed shear nonlinear hysteretic behavior and shear stress induced anisotropic effects, and (2) to reflect the strong dependency of the shear dilatancy on the effective stress ratio in both cohesionless and cohesive soils. Accurate simulation of shear-induced plastic dilation and of hysteretic effects under cyclic loading, together with full coupling between solid and fluid equations, allows capturing the build-up and dissipation of pore-water pressures and modelling the gradual softening and hardening of soil materials.

The required constitutive model parameters can be derived from the results of conventional laboratory (e.g., triaxial, (b)

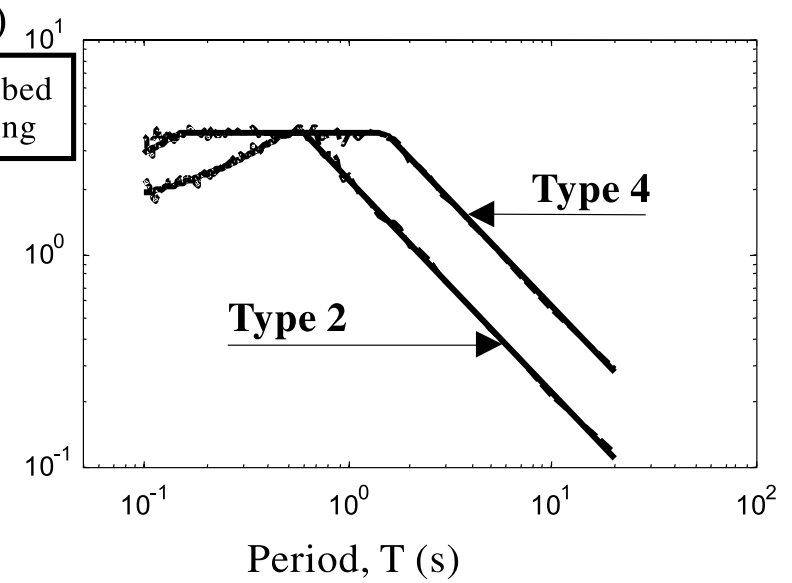

simple shear) and in situ (e.g., standard penetration, cone penetration, wave velocity) soil tests. Liquefaction strength analysis (e.g., Popescu 1995; Popescu et al. 1997) is also needed for saturated materials subjected to cyclic loads. The multiyield plasticity soil constitutive model, its implementation algorithm, and the methodology for estimating the constitutive model parameters have been repeatedly validated in the past for soil liquefaction computations, based on both centrifuge experimental results (e.g., Popescu and Prevost 1993, 1995) and full scale measurements (e.g., Keane and Prevost 1989; Popescu et al. 1992, 1998). 
Fig. 3. Arias intensities $(a)$, and number of representative cycles at $0.65 a_{\max }(b)$, computed from the acceleration time histories presented in Figs. $1 b-1 e$.

(a)

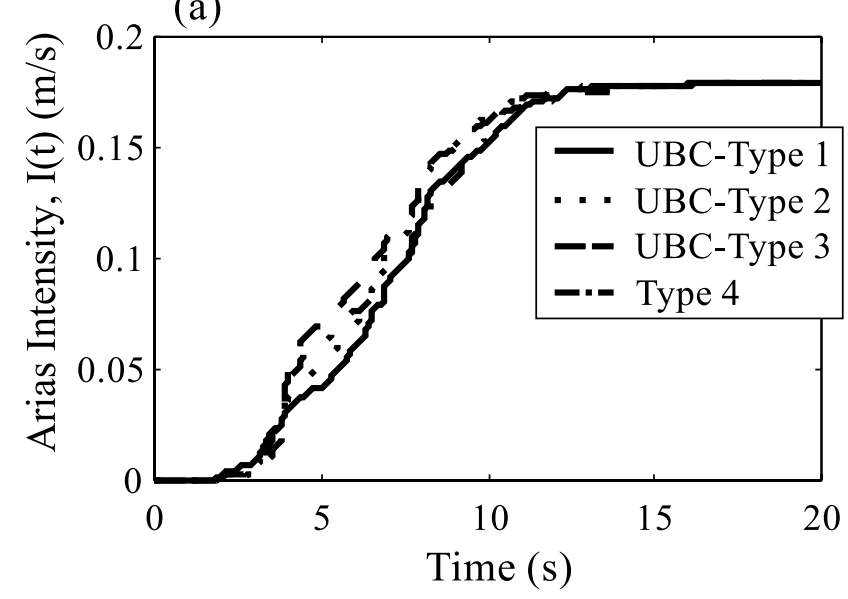

The finite element analysis is performed in one run consisting of two steps. First, gravity loads are applied and the soil is allowed to fully consolidate. The consolidation phase is calculated dynamically, by setting the Newmark algorithm parameters in the integration scheme as $\gamma=1.5$ and $\beta=1$. After consolidation is completed, the nodal displacements, velocities, and accelerations are zeroed, the time is reset to zero, and the input acceleration is applied at the base. The Newmark parameters are chosen as $\gamma=0.65$ and $\beta=(\gamma+$ $0.5)^{2} / 4=0.33$. This choice for $\gamma$ introduces a slight numerical damping ( $\gamma=0.5$ corresponds to no numerical damping), and the selected value for $\beta$ maximizes high frequency numerical dissipation. No additional viscous damping is introduced.

\subsection{Example 1: liquefaction assessment of a soil deposit}

\subsection{Finite element model}

Liquefaction risk assessment of a saturated soil deposit is next presented to illustrate the influence of the seismic loading rate. A loose to medium dense sand deposit, with geomechanical properties as well as spatial variability characteristics estimated from the piezocone test results presented in Fig. 4, is subjected to a horizontal earthquake motion, as shown in Fig. 5a. Response spectrum compatible acceleration time histories are used, considering two possible situations (1) an acceleration time history compatible with the UBC Type 2 response spectrum (Fig. 1c) corresponding to the presence of a relatively stiff soil layer underlying the sand deposit under consideration, and (2) an acceleration time history compatible with the UBC Type 4 response spectrum (Fig. 1e) that may be characteristic of a site that is close to the epicenter. The frequency ranges of the maximum spectral values are (Fig. 1a) between 1.8 and $6.7 \mathrm{~Hz}$ for Type 2 input motion, and between 0.7 and $2 \mathrm{~Hz}$ for Type 4 input motion. The seismic acceleration is prescribed at the base nodes of the finite element mesh. A semiinfinite horizontal soil deposit is simulated by imposing the same displacements in both horizontal and vertical directions, and for both solid and fluid phases, at the lateral nodes situated in the same horizontal planes (same elevations).

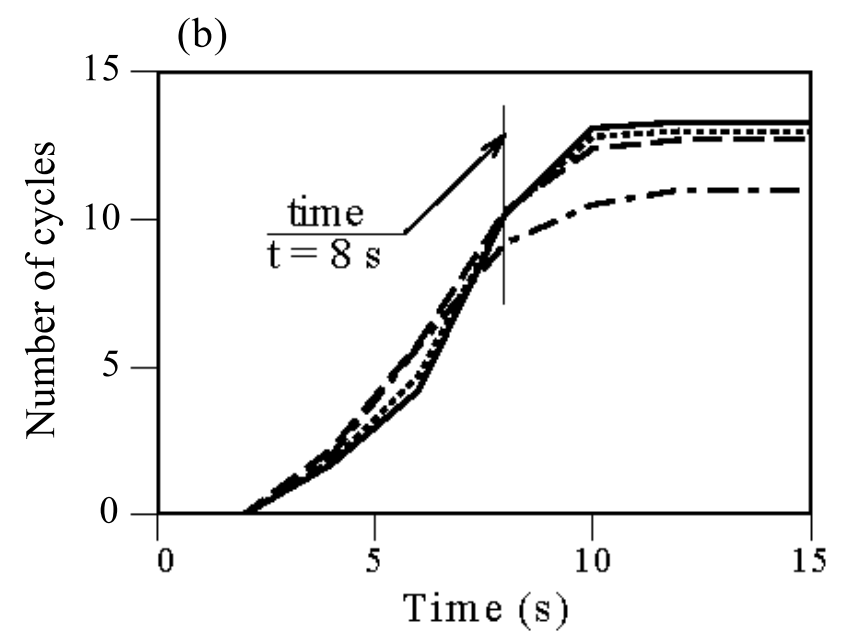

The cone penetration test results shown in Fig. 4 come from an artificial island in the Canadian Beaufort Sea (Gulf 1984). Though measured in a supposedly homogeneous man-made soil deposit, the recorded cone tip resistance shows significant spatial variations. To account for this variability, Monte Carlo simulations are performed, with soil parameters corresponding to 25 sample functions of a stochastic vector field with probabilistic characteristics derived from the field data shown in Fig. 4. Each sample function represents a possible realization of the spatial distribution of the field test results over the analysis domain. For each sample function, and at each spatial location, the simulated values of field test results are used to estimate the constitutive parameters of the soil constitutive model used for stochastic input finite element analyses. For a detailed description of the Monte Carlo simulation methodology and the estimation of the soil constitutive parameters, the reader is referred to Popescu (1995) and Popescu et al. (1997). It is mentioned that a larger number of simulations is required to provide the statistics of the response by Monte Carlo simulations. On the one hand, non-linear dynamic finite element analyses are computationally expensive, on the other hand, this study is not aimed at providing the statistics of the response, but only the general trends, as shown in the next section.

\subsection{Analysis results}

Some of the results are summarized in Fig. 5 in terms of the excess pore-water pressure (epwp) ratio with respect to the initial effective vertical stress (IEVS) and horizontal displacements at the ground level. The predictions of the Monte Carlo simulations are represented in Fig. 5 as ranges of results obtained from 25 sample functions. For comparison purposes, the predicted epwp ratio is presented in terms of: (1) Liquefaction index (Fig. $5 b$ ), computed by averaging the predicted epwp ratio in the horizontal direction (Ohtomo and Shinozuka 1990)

$$
Q(z, t)=\frac{1}{L} \int_{0}^{L} r(x, z, t) \mathrm{d} x
$$

where 
Fig. 4. Cone tip resistance recorded in a hydraulic fill deposit at Tarsiut P-45 (data from Gulf 1984).

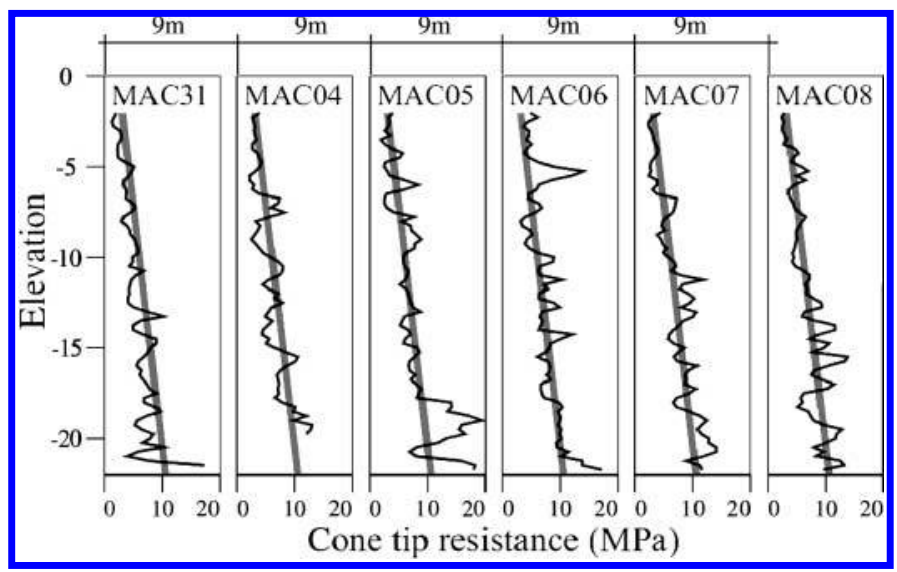

$$
r(x, z, t)=\frac{u(x, z, t)}{\sigma_{v 0}^{\prime}(x, z)}
$$

is the ratio of the epwp with respect to the initial effective vertical stress, and $L$ is the dimension of the analysis domain in the horizontal direction (Fig. 5a). At each time instant, $t$, the liquefaction index, $Q(z, t)$, characterizes the distribution of the epwp ratio in elevation.

(2) Area of liquefied zone, or $A_{80}$ index (Fig. $5 c$ ), defined as (Popescu et al. 1997)

$$
A_{80}(t)=\frac{1}{L H} \int_{0}^{H} \int_{0}^{L} 1_{[r(x, z, t) \geq 0.8]} \mathrm{d} x \mathrm{~d} z
$$

where $H$ is the depth of the saturated soil layer (Fig. 5a); and $1_{[P]}=1$ if $P$ is true, and $1_{[P]}=0$ if $P$ is false. Thus, at each time instant, the $A_{80}$ index represents the proportion of the saturated soil deposit for which the epwp ratio, $r$, exceeds $80 \%$.

Comparisons in terms of predicted horizontal displacements at the ground level and their evolution with time are provided in Fig. $5 d$. Differential displacements between the ground level and the base of the analysis domain are considered, as shown below

$$
\delta_{\mathrm{h}}(t)=\delta^{\text {ground level }}(t)-\delta^{\text {base }}(t)
$$

For the soil deposit analyzed, significantly larger pore pressures and horizontal displacements are predicted by the numerical model when using the Type 4 input motion, with lower frequency content, than for the Type 2 input motion (Fig. 5). The discrepancy between the results obtained for the two types of input motions becomes larger with time, as illustrated in Fig. $5 c$. Gradual build-up of epwp leads to softening of the soil (decrease of shear modulus and shear strength) and subsequent changes in the vibration characteristics. The characteristic frequency of the soil deposit gradually decreases to values that are closer to the frequency range corresponding to the Type 4 response spectrum. Therefore, the Type 4 input motion transmits more energy to the soil deposit than the Type 2 input motion.

The results in terms of the epwp ratio obtained at three different time instants for one of the sample functions used in the Monte Carlo simulations are presented in Fig. 6. As observed, liquefaction is predicted to occur in patches corre- sponding to the location of loose pockets in the heterogeneous soil mass. For further details and results obtained for other sample functions of assumed soil variability, the reader is referred to Popescu et al. (1997). For the case presented in Fig. 6, significant differences can be observed between the epwp build-up predicted for the same soil deposit and using input seismic motions with the same energy, but with different frequency ranges of the maximum spectral values.

A simplified liquefaction assessment procedure (e.g., Das 1983) was used by Popescu et al. (1997) for the soil deposit presented in this example. The procedure currently adopted by geotechnical practice is based on empirical correlations between observed liquefaction resistance under ground level conditions and normalized penetration resistance (SPT blow count or cone tip resistance). Using the average values of recorded cone tip resistances plotted in Fig. 4 and the number of representative cycles, $N_{\mathrm{c}}$, estimated for the acceleration time histories in Figs. $1 b-1 e$, it was predicted that after $8 \mathrm{~s}$ of shaking, corresponding to $N_{\mathrm{c}}=10$ (Fig. $3 b$ ), the soil would liquefy at a depth between 3 and $12 \mathrm{~m}$. The numerical results in terms of the liquefaction index presented in Fig. $5 b$, predict liquefaction at a depth between 4 and $7 \mathrm{~m}$ for the Type 2 input, and between 4 and $10 \mathrm{~m}$ for the Type 4 input. Accounting for the fact that the simplified procedure for liquefaction assessment is conservative (CPT based liquefaction boundary curves correspond to a probability of liquefaction $P_{\mathrm{L}}=50 \%$, see e.g., Juang and Jiang 2000) the numerical results presented in Figs. 5 and 6 seem realistic. The current practice approach however, is not able to differentiate between seismic motions with different loading rates (e.g., for the case at hand, the higher frequency input produced considerably less liquefaction).

\subsection{Example 2: seismic analysis of an embankment dam}

\subsection{Finite element model}

In this numerical example, the effects of frequency content of the input motion are illustrated based on results of the seismic evaluation of an embankment dam. The cross section of the dam is presented in Fig. 7. The earthfill body consists of permeable upstream (U/S) and downstream (D/S) shells, with average slopes of about 3.5:1, and an impervious clay-core. The soil material properties for the dam body are selected to be relatively resistant to soil liquefaction. The foundation consists mainly of soft to medium stiff clays and dense sands. There is however, a layer of potentially liquefiable silty sand located at a depth of about 6-10 $\mathrm{m}$ and extending over the entire length of the dam. The dimensions, layout, and zonation of the embankment dam used in this example are fictitious, however the properties of the foundation soils have been estimated based on real in situ standard penetration and cone penetration test results.

Two-dimensional (plane strain) nonlinear dynamic finite element analyses are performed for the cross section of the dam shown in Fig. 7 using the code DYNAFLOW (Prevost 1999). The soil situated above the water level is discretized into quadrilateral solid finite elements and the saturated soil into quadrilateral two-phase medium elements with four degrees of freedom (d.o.f.) per node (two for the solid and two for the fluid phase displacements). The finite element mesh 
Fig. 5. Liquefaction strength assessment of a soil deposit. The results in $(b),(c)$, and $(d)$ represent ranges of predictions from Monte Carlo simulations accounting for spatial variability of soil properties.

(a) Soil deposit analyzed in Example 1 (the soil characteristics correspond to a hydraulic fill deposit - in situ recorded cone tip resistances are shown in Fig. 4)

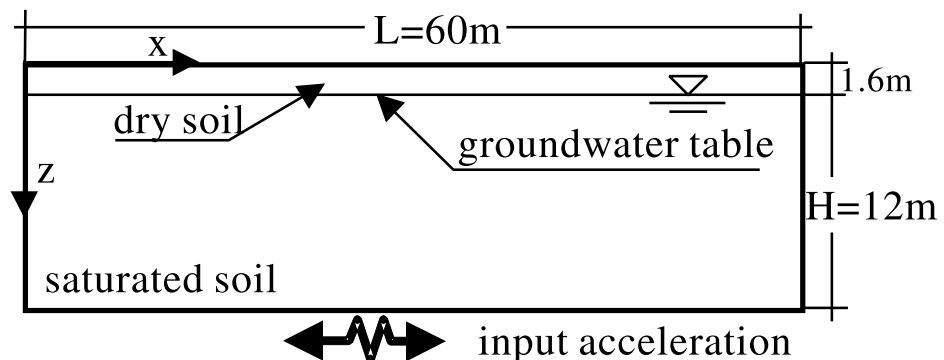

(b) Liquefaction index at time $\mathrm{t}=8 \mathrm{~s}$

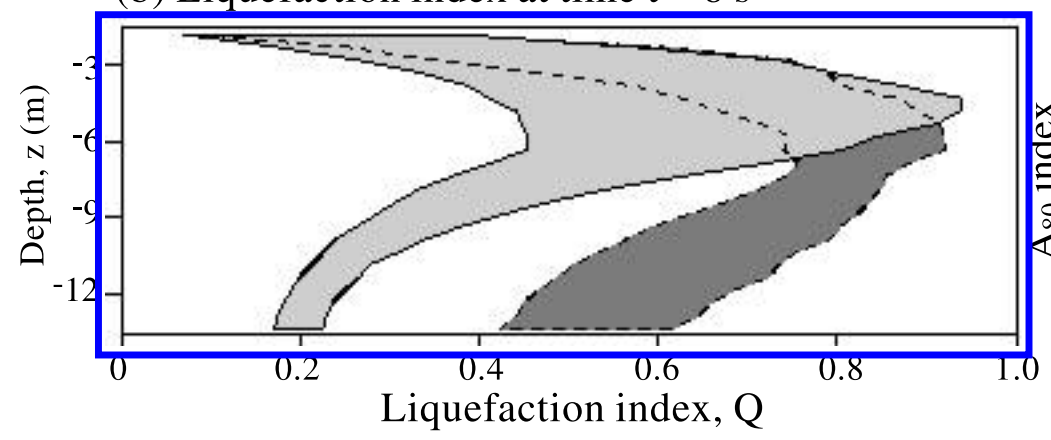

(d) Horizontal displacements at the ground level

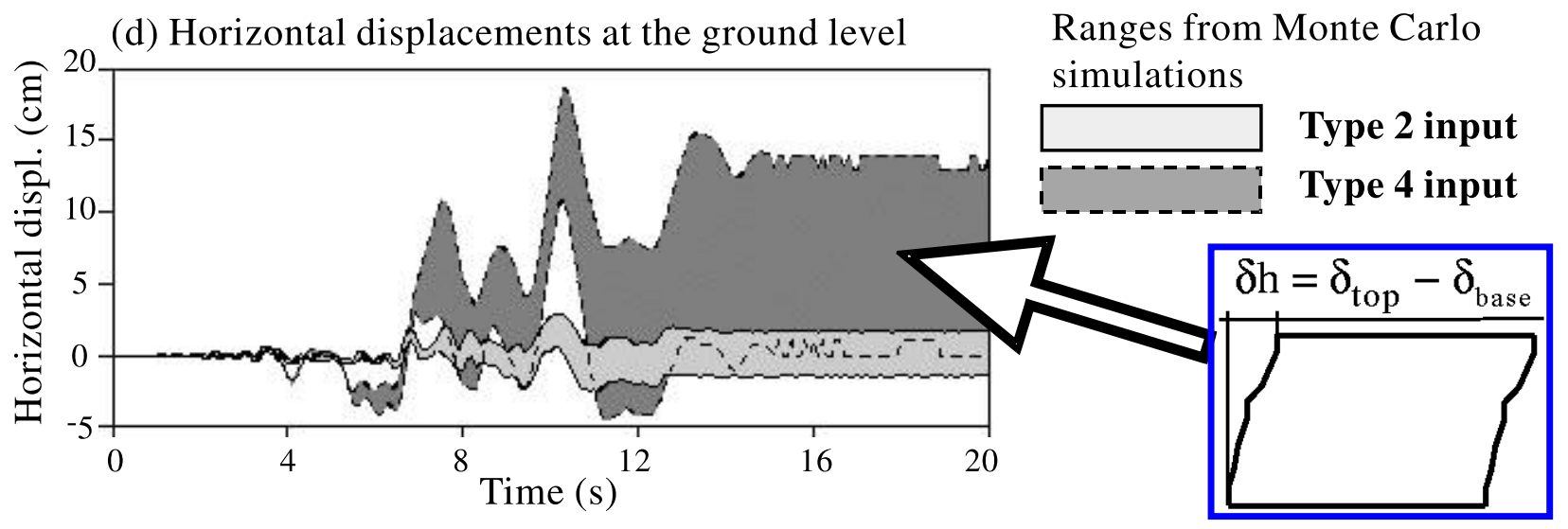

is extended vertically in the foundation down to an assumed impervious soil layer. The analysis domain is extended laterally about $250 \mathrm{~m}$ upstream and downstream of the dam toes to avoid the influence of the lateral boundary conditions on the numerical results obtained for the zone of interest. The boundary conditions are prescribed as follows: (1) no vertical motion at the base for both solid and fluid phases, (2) prescribed acceleration to the solid phase horizontal d.o.f. at the base nodes, and (3) prescribed free field motion to the lateral nodes of the foundation (the procedure is described in Popescu et al. 1998). The analysis is performed in one run, as described in Section 3, and includes soil consolidation, stepby-step dynamic analysis, and postearthquake behaviour.

Three of the acceleration time histories presented in Fig. 1 are selected as base input motions. The first one is compatible with the UBC Type 1 response spectrum (Fig. 1b) and has a frequency range of the maximum spectral values between 2.6 and $6.7 \mathrm{~Hz}$. The second is compatible with the UBC Type 3 response spectrum (Fig. 1d) and has a frequency range of the maximum spectral values between 1.1 (c) Area of liquefied zone

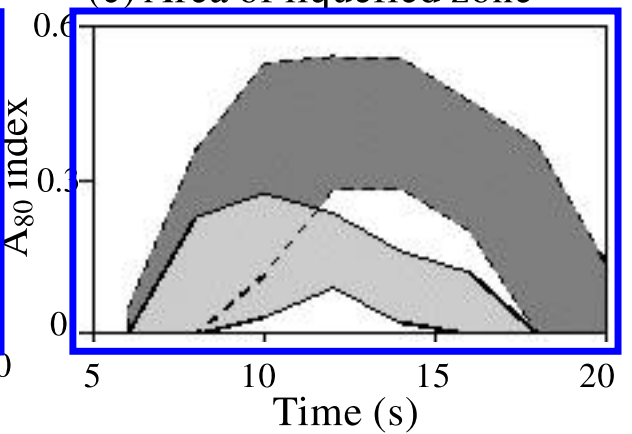

Ranges from Monte Carlo simulations

and $5 \mathrm{~Hz}$. The third (Fig. 1e) is compatible with the fictitious acceleration response spectrum labeled "Type 4" in Fig. $1 a$, and has a frequency range extended more towards lower values $(0.7-1.6 \mathrm{~Hz})$. To ensure visible seismic structural damage, the acceleration time histories shown in Figs. $1 b, 1 d$, and $1 e$ have been scaled to a maximum value of $0.3 g$ in this numerical example. To assess possible postearthquake effects, the analysis period has been extended beyond the duration of the earthquake (analysis time $=30 \mathrm{~s}$ ).

\subsection{Predicted excess pore pressures}

A comparison between predicted excess pore-water pressure (epwp) build-up at the end of the strong shaking period (time $t=12 \mathrm{~s}$ ) for the three cases analyzed is presented in Fig. 8. The numerical results are shown as contours of epwp ratio with respect to the initial effective vertical stress (IEVS). Very large pore pressures and soil liquefaction are predicted in the loose silty sand layer in the vicinity and below the dam toes for all three cases analyzed. No epwp build-up is predicted in the dam body, which consists of liq- 
Fig. 6. Contours of excess pore pressure ratio predicted for the soil deposit in Fig. $5 a$ using base input accelerations with different frequency content (the material properties have been assessed according to one of the sample functions used for the Monte Carlo simulations).

Base input acceleration compatible with:

(a) Type 2 response spectrum (b) Type 4 response spectrum
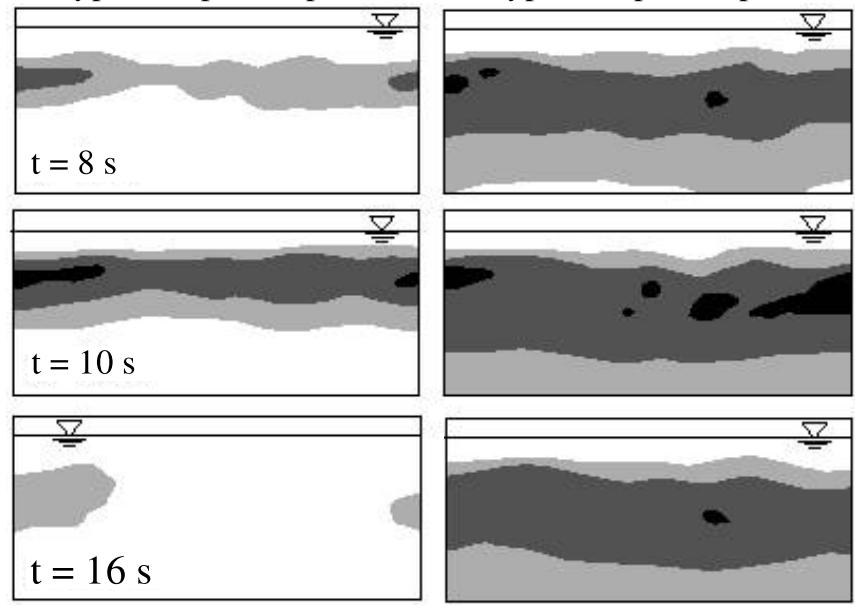

Grey scale range for the epwp ratio $\left(\mathrm{u} / \sigma_{\mathrm{v} 0}\right)$

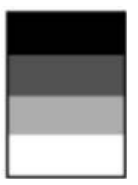

1.00

0.90

0.70

0.50

0.00

uefaction resistant materials. The epwp predicted in the loose silty sand layer below the dam core have reduced values due to large static shear stresses and overburden stresses in this area. These results are in good agreement with field observations (e.g., Seed and Harder 1990) and recent centrifuge experimental results (Steedman et al. 2000).

The evolution of predicted epwp ratio at three locations in the silty sand layer is presented in Fig. 9. During the strong shaking period, very similar values are predicted below the dam toes (Figs. $9 a$ and $9 c$ ) for all three cases analyzed. After the shaking, the pore pressures dissipate in the case of Type 1 input motion, while for Type 3 and 4 inputs they continue to increase. An explanation can be found in continuous postearthquake shear deformations, through a mechanism similar to static liquefaction. Predicted values of the epwp ratio with respect to the IEVS for Types 3 and 4 seismic inputs exceed unity below dam toes. This is because of the predicted slope failures that modify the total vertical stress at those locations. Some dilation is predicted after the end of strong shaking below the D/S toe for the Type 1 input motion.

The pore pressures predicted in the loose silty-sand layer below the core (Fig. 9b) are larger for the Type 3 and Type 4 seismic inputs than for the Type 1 input. However, no liquefaction is predicted at this location. Excess pore pressure dissipation after the shaking is predicted at this location for all three cases, due to the effect of large confining stresses.

The numerical model predictions in terms of epwp ratio in the loose silty sand layer below the dam core are compared in Fig. $9 b$ with the results of a centrifuge experimen- tal study on the behaviour of saturated sands under high effective confining pressure (Steedman et al. 2000). A large number of tests on saturated loose sands under IEVS of up to $1000 \mathrm{kPa}$ and subjected to earthquake-like shaking have been performed. The base input motion used in the centrifuge experiments had a frequency of about $1 \mathrm{~Hz}$ (at the prototype scale), which is within the range of the Type 3 and Type 4 input motions used in this study. The experimental results indicated that, at high IEVS and under moderate amplitudes of excitation, the maximum level of epwp ratio with respect to the IEVS is capped at values below $100 \%$ (i.e., the soil does not reach initial liquefaction). For example, for IEVS of about $600 \mathrm{kPa}$, the maximum epwp ratios recorded in the centrifuge study ranged between $40 \%$ and $60 \%$. The maximum epwp predicted in the loose silty sand layer below the dam core for Type 3 and Type 4 inputs are in good agreement with those experimental results (Fig. 9b).

\subsection{Predicted deformations}

Predicted maximum shear strains and deformed shapes of the dam are presented in Fig. 10 for the three cases analyzed. At the end of the strong shaking period (time $t=12 \mathrm{~s}$, Figs. $10 a-10 c)$, the finite element model predicts a certain degree of damage at the $\mathrm{D} / \mathrm{S}$ toe for all three cases. This damage is due to very large shear deformations in the silty sand layer and local slope failure of the dam toe. Large shear deformations are also predicted below the U/S toe for input motion Types 3 and 4 . In general, the numerical model does not predict significant differences in terms of deformations at the end of shaking for the three cases analyzed

Figures $10 d-10 f$ present predicted maximum shear strains and deformed shapes of the dam at the end of analysis (time $t=30 \mathrm{~s}$ ). The differences between the three cases analyzed are now very significant. Except for a limited zone at the D/S toe affected by relatively large shear deformations, no significant damage (or at least, no progressive failure) is predicted for the dam when the Type 1 acceleration time history is used as input motion (Fig. 10d). The large deformations predicted at the $\mathrm{D} / \mathrm{S}$ toe do not seem to immediately endanger the overall safety of the dam. In the case of the input acceleration time histories compatible with the Type 3 and Type 4 response spectra, the numerical model predicts postearthquake failure of the dam. Failures of both the U/S and the D/S slopes, extending from toe to crest, are predicted in the case of Type 3 input motion (Fig. 10e). The slope failures occur after the end of strong shaking and continue at relatively constant speed after the earthquake to the end of the analysis period. For this input motion, the numerical model does not predict direct damage to the core during the analysis period. General failure of the dam is predicted in the case of Type 4 input motion (Fig. 10f). As in the previous case, the U/S and D/S slopes exhibit progressive failure after the end of strong shaking. By the end of analysis (time $t=30 \mathrm{~s}$ ), the failure of the U/S shell extends into a general failure of the dam, with a slide surface developing through the core.

Predicted displacement time histories are plotted in Fig. 11 for the U/S zone of the crest. Relatively small horizontal displacements (about $20 \mathrm{~cm}$ ) and settlements (about $40 \mathrm{~cm}$ ) are predicted when using the Type 1 input. These 
Fig. 7. Seismic analysis of an embankment dam: cross section showing the soil materials and the finite element mesh. U/S, D/S, upstream and downstream, respectively.

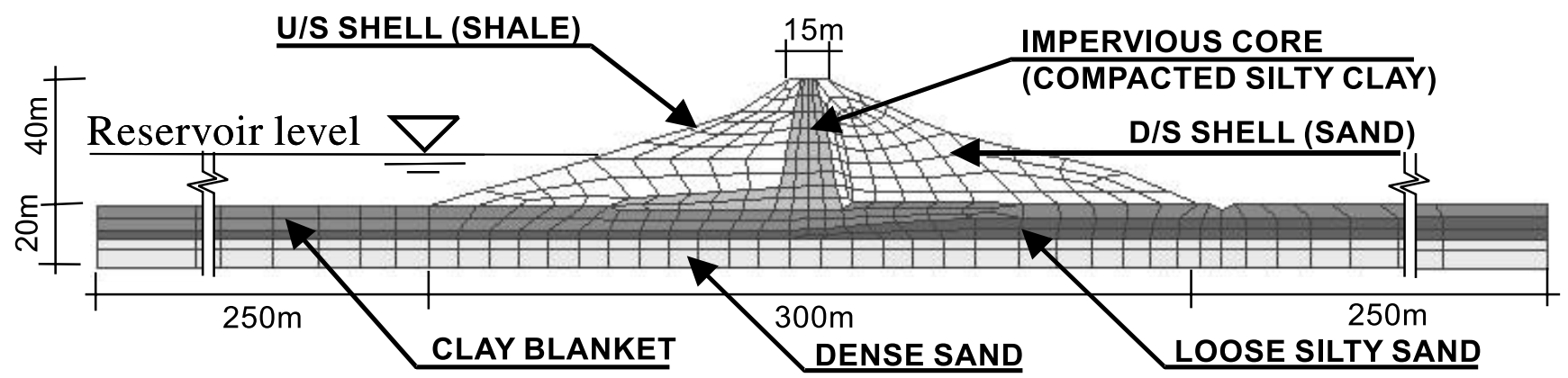

Fig. 8. Seismic analysis of an embankment dam: predicted contours of excess pore-water pressure (epwp) ratio with respect to the initial effective vertical stress (IEVS).

END OF STRONG SHAKING $($ time $=12 \mathrm{~s})$

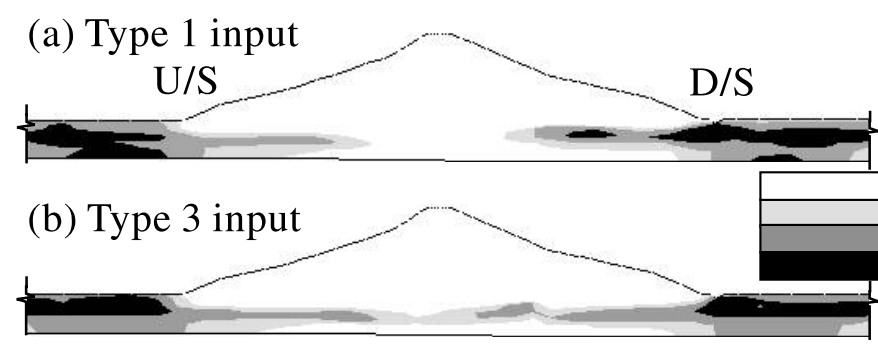

(c) Type 4 input

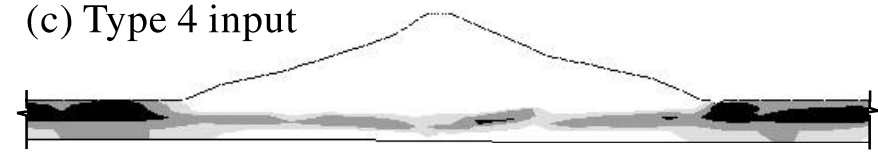

displacements occur during the strong shaking period (from time $t=4 \mathrm{~s}$ to $t=12 \mathrm{~s}$ ), and then stabilize. In the case of Type 3 and Type 4 input motions, the predicted crest displacements are significantly larger (1-4 $\mathrm{m}$ at the end of shaking, and much larger at the end of analysis). For these cases, the displacements are predicted to continue at a relatively constant speed after the end of shaking, indicating progressive slope failures.

\subsection{Discussion}

The fact that very different structural responses are predicted for the three seismic inputs, even if the epwp distributions at the end of strong shaking are similar, could be explained by analyzing the evolution of the vibration characteristics of the dam-foundation system during and after the shaking. The characteristic period (corresponding to the first mode of vibration of the structure) and its evolution with time is presented in Fig. 12a. It was calculated by the finite element program using eigenvalue analysis after the consolidation phase, and then every $0.5 \mathrm{~s}$ during the step-by-step dynamic analysis, as a function of the current state of the structure (e.g., values of the shear moduli).

Under static conditions (before the earthquake), the damfoundation system has a period of vibration of about $1 \mathrm{~s}$. This relatively large value is due to the presence of the soft to medium clay and loose silty sand layers in the foundation. As epwp start building up in the silty sand, this layer softens
END OF ANALYSIS (time = $30 \mathrm{~s}$ )
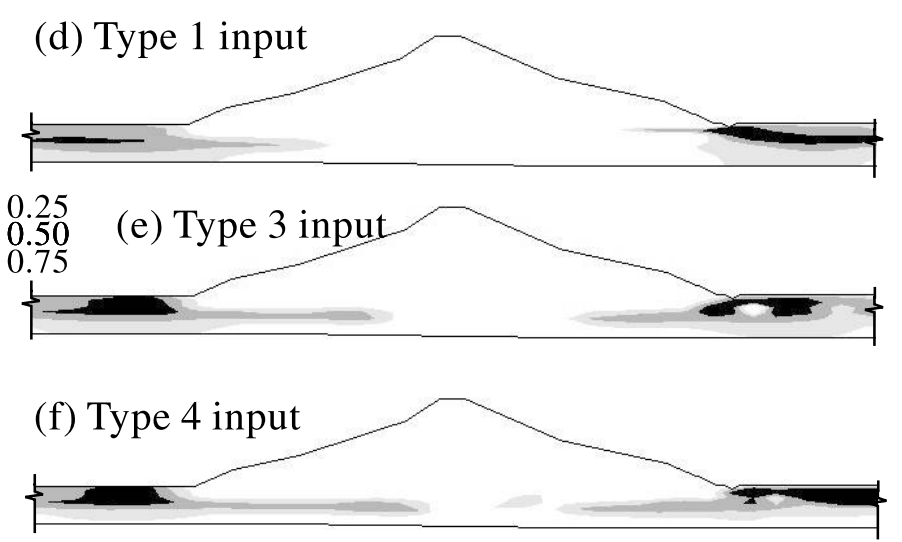

and the dam is practically "floating" on the foundation. This leads to very large vibration periods (about $2.5 \mathrm{~s}$ ) during the strong shaking period. With epwp dissipation, the silty sand layer partially regains its shear strength and the characteristic period of vibration decreases. This decrease is visible for Type 1 input motion and insignificant for the other two cases, where epwp dissipation is prevented by continuing large deformations.

From the evolution of the epwp ratio (Fig. 9) and of the characteristic period of vibration (Fig. 12a) during the first part of the earthquake, it can be concluded that initially all three types of input motion have similar effects on the structure. After the vibration period increases (at time $t=8 \mathrm{~s}$ ), the three input motions start delivering significantly different amounts of energy. This fact is illustrated in Fig. 12b, where the spectral amplitudes for Type 1 and Type 4 response spectra are compared at a characteristic period $\mathrm{T}=2 \mathrm{~s}$. It can be concluded from this comparison that for a structure with a characteristic period of about $2 \mathrm{~s}$, Type 4 input motion delivers significantly more energy per unit mass than Type 1 input motion. For example, for a 1 d.o.f. structure having a period of vibration $\mathrm{T}=2 \mathrm{~s}$, the energy delivered by a Type 4 input would be 16 times larger than the one delivered by a Type 1 input. This fact explains the dramatic differences in the seismic effects predicted for the dam-foundation system using three input accelerations that only differ in frequency content. 
Fig. 9. Seismic analysis of an embankment dam: evolution with time of the predicted excess pore-water pressure (epwp) ratio at three locations within the loose silty sand layer: $(a)$ below the $\mathrm{U} / \mathrm{S}$ toe; $(b)$ below the core; $(c)$ below the D/S toe. Legend for all plots in Fig. 9c. IEVS, initial effective vertical stress.
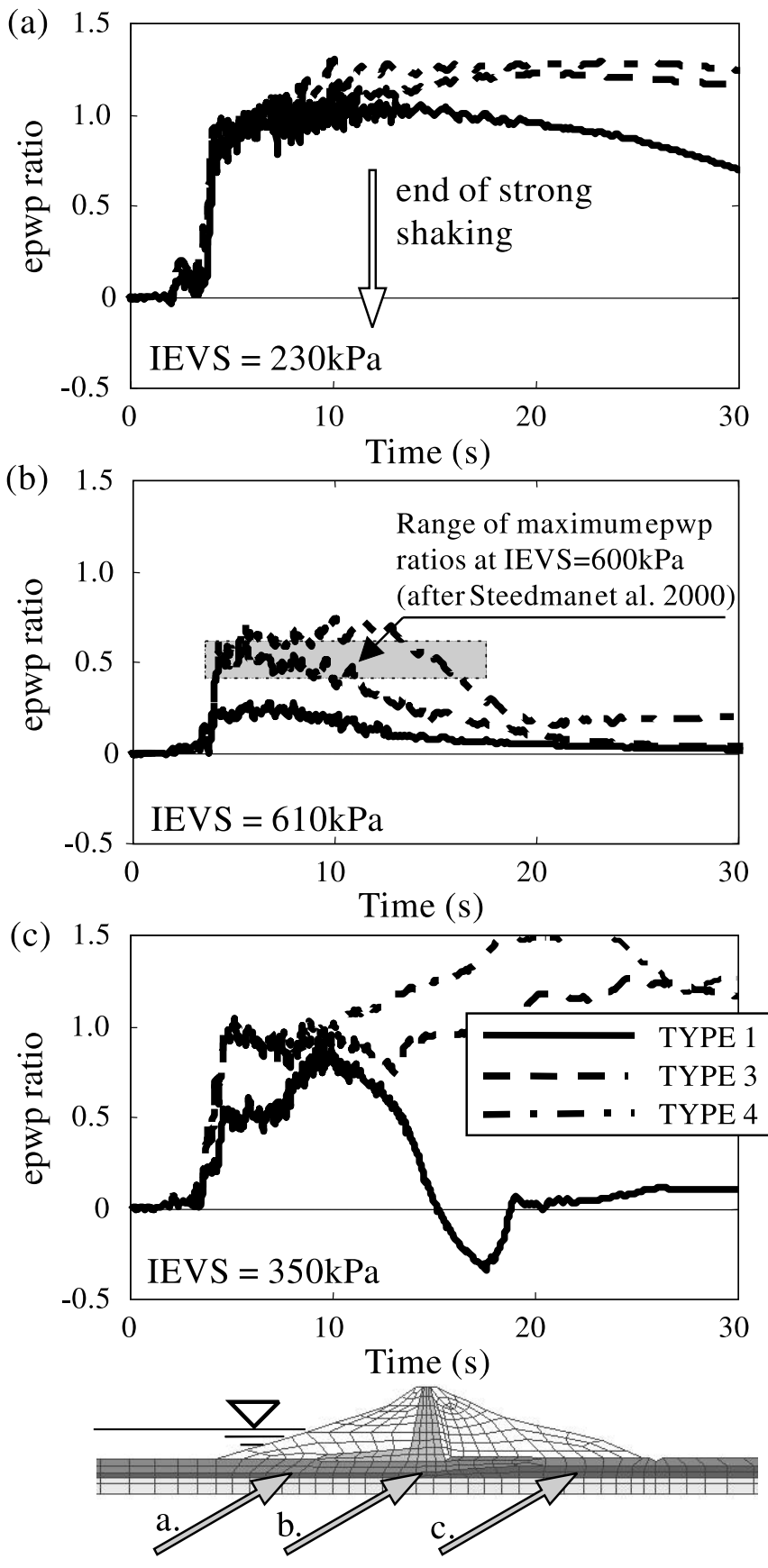

\subsection{Variability of the structural response}

Results obtained from only one simulation have been presented so far for each type of seismic input. To verify if these results are representative, i.e., if there is indeed significant variation in the structural response induced by differences in seismic loading rate, results of a study on structural response variability induced by seismic accelerations representing various sample functions of the same stochastic process are presented in this section. To this end, 20 sample functions were digitally generated, 10 of them compatible with the Type 1 response spectrum and the other 10 compatible with the Type 3 response spectrum. All of the acceleration time histories are shown in Fig. 13. The sample function referred to as "sample \#1" in Fig. 13a represents the acceleration time histories compatible with Type 1 and Type 3 response spectra that were used throughout this numerical example and are shown in Figs. $1 b$ and $1 d$.

The acceleration time histories were generated using the procedure presented in Section 2.1, as explained hereafter. The simulation procedure, based on the spectral representation method, employs one set of uniformly distributed random phase angles for each resulting sample function. Use of different "seed numbers" produces different sets of random phase angles and therefore different resulting sample functions of seismic acceleration. These sample functions have the same probabilistic characteristics and are scaled to represent earthquakes delivering the same amounts of energy (they have the same Arias Intensity). As for the number of sample functions (10 for each type), it is certainly too small to allow any statistical inference on the resulting structural response. It is deemed however, that for the purposes of this study 10 samples are sufficient to infer the general trends and ranges of the structural response when the structure is subjected to seismic motions having different frequency contents (namely Type 1 and Type 3).

Predicted maximum shear strains and deformed shapes of the dam at the end of analysis (time $t=30 \mathrm{~s}$ ) are presented in Fig. 14 for all samples of input acceleration. The results presented in Fig. 14a (sample input \#1) are the same as those in Figs. $10 d$ and $10 e$. It clearly results from Fig. 14 that there are two distinct types of structural response, corresponding to two different types of seismic motion. When subjected to seismic motions compatible to the Type 1 response spectrum the dam does not fail. Large deformations are predicted both at the U/S toe (slumping of the bottom third of the slope) and at the D/S toe (rotational failure of the lower one half to two thirds of the slope), but the overall structural safety of the dam is not immediately compromised. When the dam is subjected to the Type 3 seismic motions, the numerical model predicts progressive failure of both the U/S and the D/S slopes in all 10 cases. Except for one input acceleration (sample input \#6, Fig. 16f) the core is not strongly affected during the analysis period.

Figure 15 presents predicted displacements at the U/S edge of the dam crest. The results obtained for 10 sample functions of seismic inputs compatible with the Type 1 acceleration response spectrum are in a very close range. For all of the cases, the predicted crest displacements are small and stabilize at the end of shaking. The final values of horizontal displacements range between 0.15 and $0.3 \mathrm{~m}$, and the settlements between 0.3 and $0.5 \mathrm{~m}$.

Except for sample \#4, the numerical model predicts similar behaviour for all of the seismic inputs compatible with the Type 3 response spectrum. The corresponding crest displacements plotted in Fig. 15 indicate failure of the U/S slope, continuing after the end of the earthquake at a relatively constant speed, indicating progressive slope failures. When the dam is subjected to the sample \#4 seismic input, the U/S end of dam crest is predicted to move less than for the other cases and to stabilize after the end of shaking. This 
Fig. 10. Seismic analysis of an embankment dam: predicted deformed shapes and contours of maximum shear strains. The deformation scale is the same as the geometric scale.

END OF STRONG SHAKING (time $=12 \mathrm{~s})$

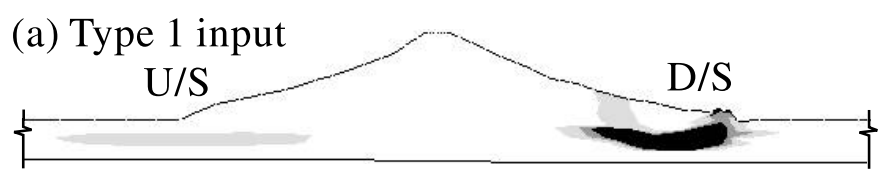

(b) Type 3 input

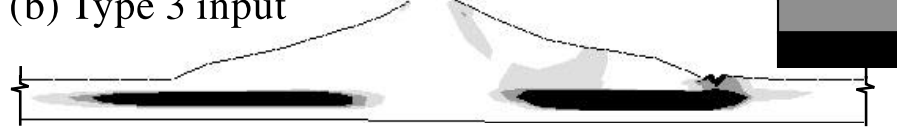

(c) Type 4 input

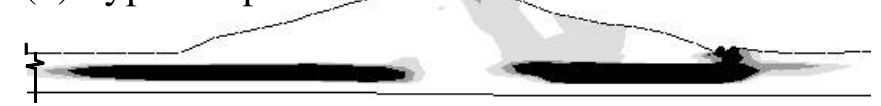

Fig. 11. Seismic analysis of an embankment dam: predicted crest displacements.
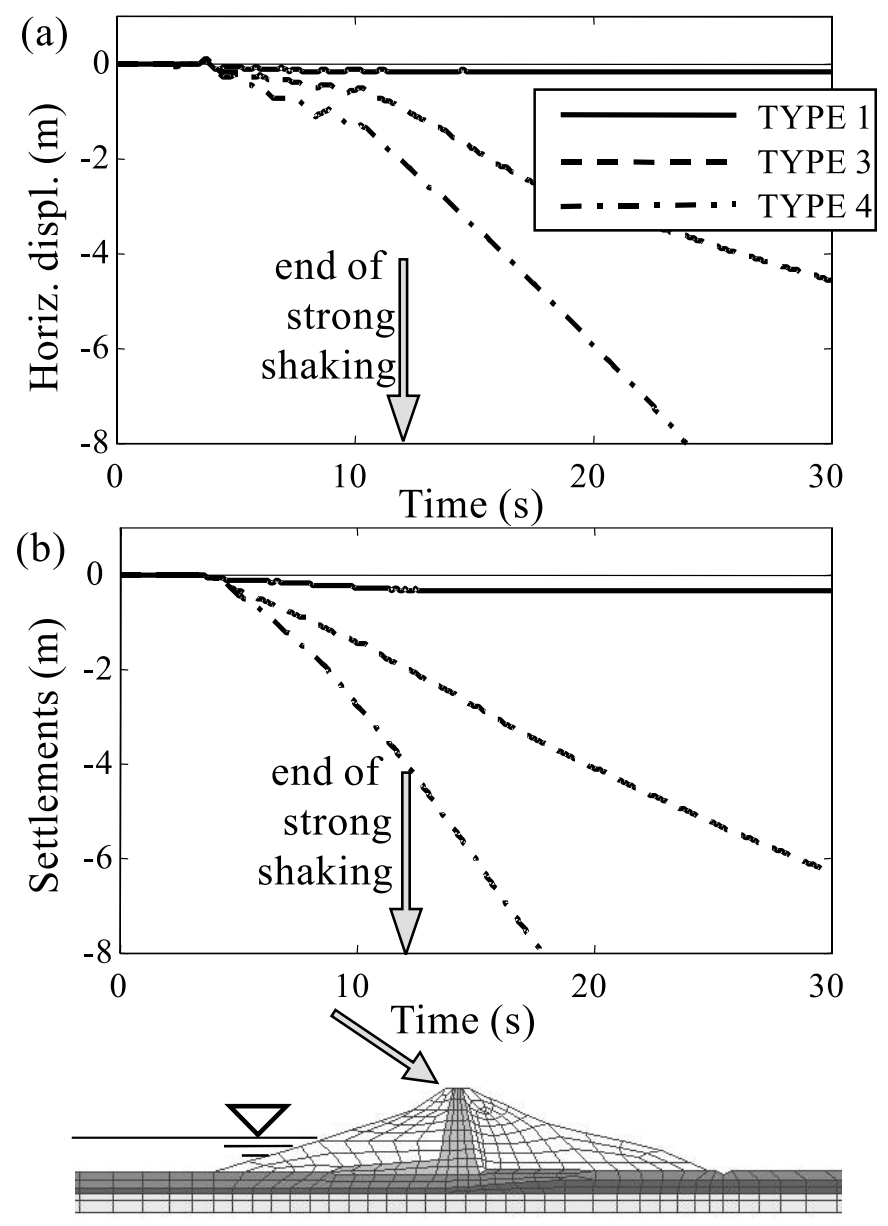

is due to the fact that the U/S slope failure is predicted to initiate below the crest for this case (see Fig. 14d).

The crest displacements predicted using the Type 4 input motion (one single sample function) are also plotted for comparison. The crest displacements predicted using 10
END OF ANALYSIS (time $=30 \mathrm{~s}$ )

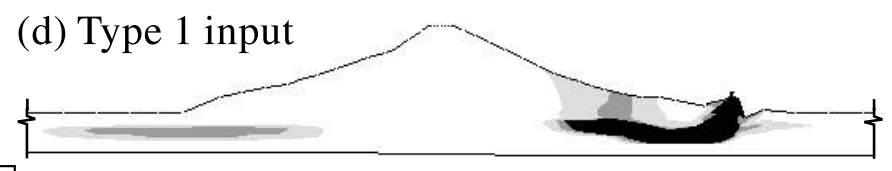

$33 \%$

$67 \%$

$100 \%$

(e) Type 3 input

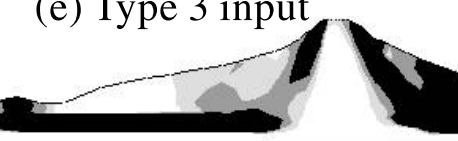

(f) Type 4 input

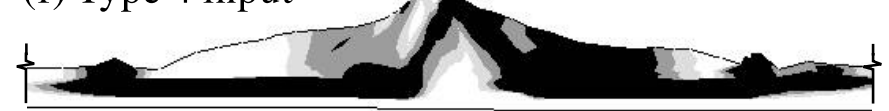

Fig. 12. Analysis of the interplay between the vibration characteristics of an embankment dam and the frequency content of the input motion: $(a)$ evolution of the characteristic period of vibration of the dam-foundation system; $(b)$ acceleration response spectra of the base input motions used in the study.
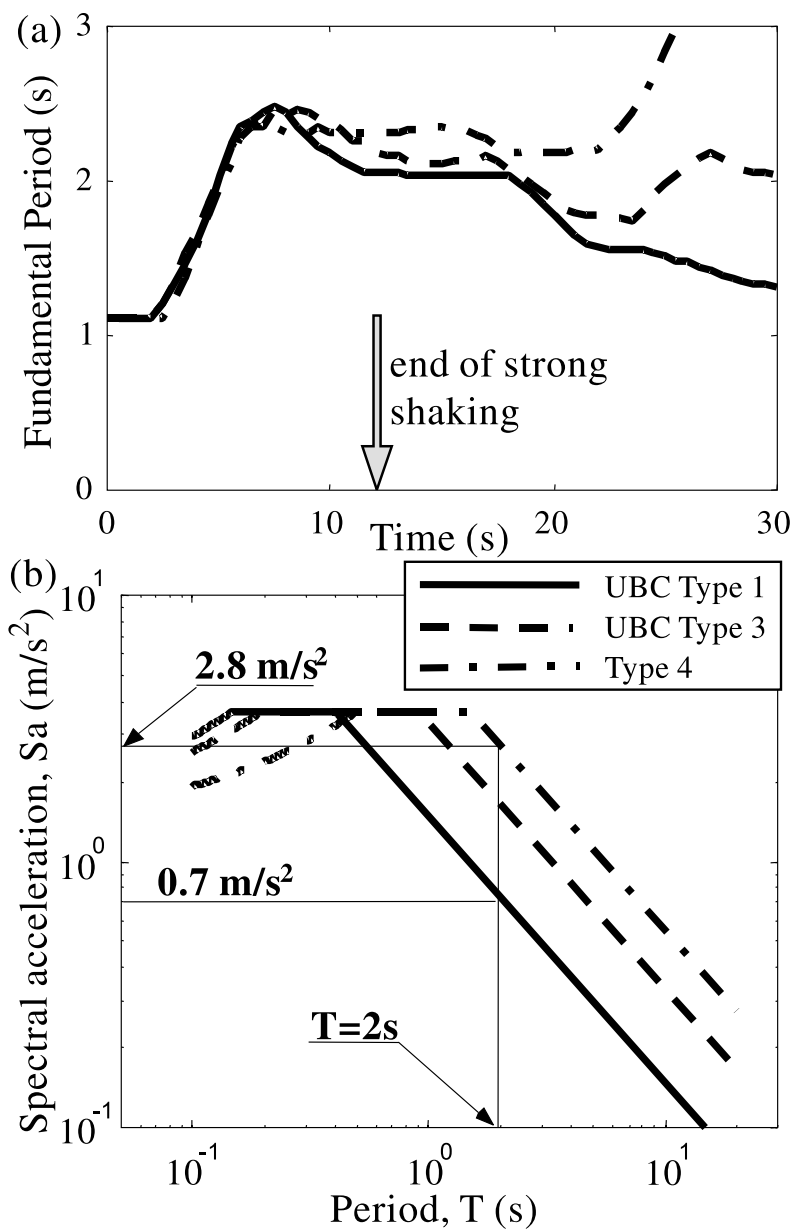

sample functions of Type 3 input motion resulted in a relatively large range, but all are contained between displacements predicted using Type 1 and Type 4 inputs. The 
Fig. 13. Ten sample functions of seismic inputs compatible with the UBC Type 1 design response spectrum (left) and with the UBC Type 3 design response spectrum (right).

TYPE 1 seismic input
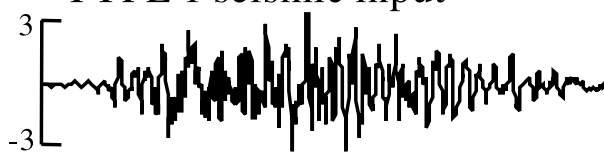

(a) Sample \#1 $1^{3}$

TYPE 3 seismic input
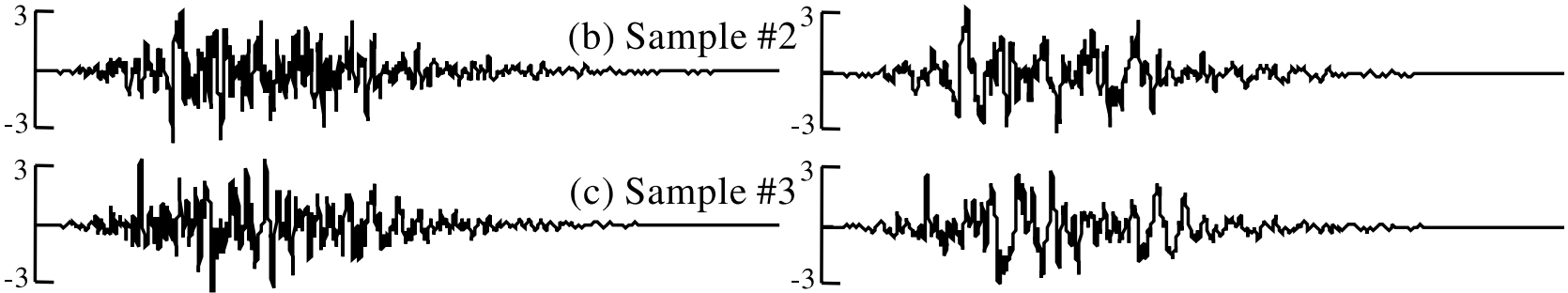

(c) Sample \#3 $3^{3}$

(Figure 1b)

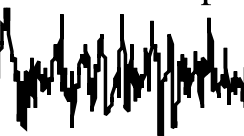

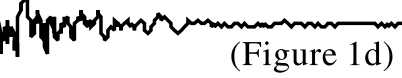
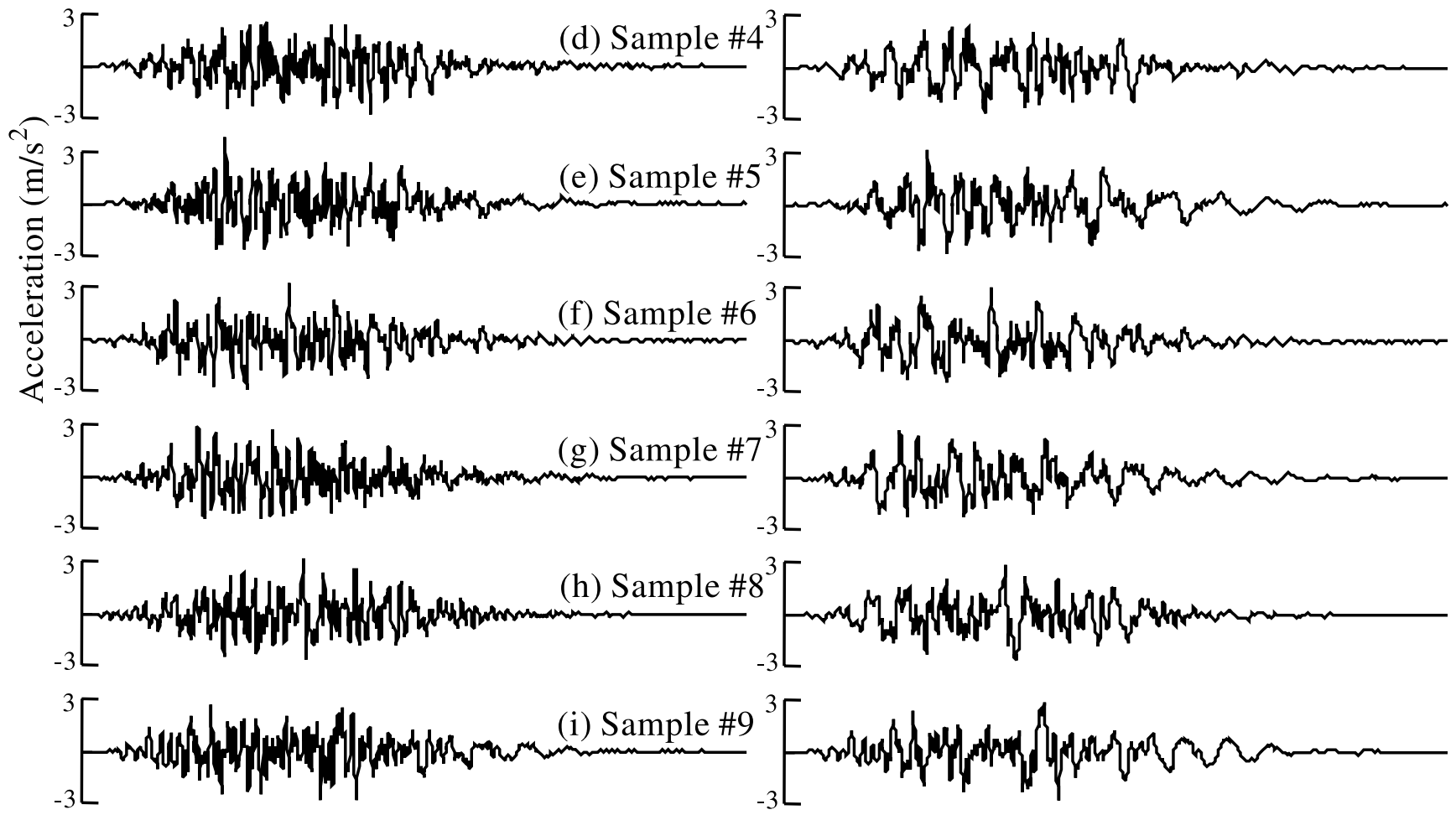

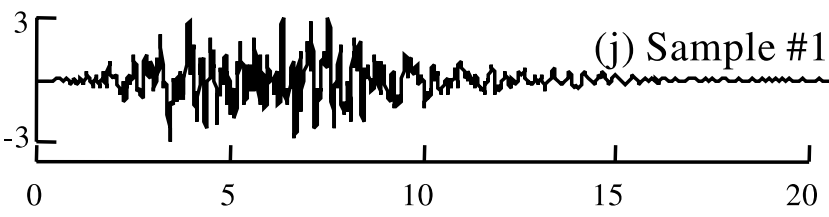

Time (s)

scatter can be explained by the occurrence during post-liquefaction deformations of rigid body motions whose magnitudes are governed by particular features of the seismic input.

\subsection{Conclusions}

To assess the effects of loading rate (frequency content of seismic excitation) on dynamic behaviour, earthquake ground motions corresponding to different local soil conditions were simulated using four different response spectra. The seismic acceleration time histories were generated as uniformly modulated nonstationary stochastic processes, in accordance with prescribed response spectra and modulating functions. They were similar in amplitude, duration of the strong ground motion, Arias intensity (which is a measure of energy delivered per unit mass), and number of representative cycles at $0.65 a_{\max }$ (which is also used as a measure of the seismic effects). The only difference among the four seismic ground motions used in this study was in the frequency ranges of the maximum spectral values.

Nonlinear dynamic finite element analyses in terms of effective stresses were carried out for a saturated soil deposit 
Fig. 14. Predicted deformed shapes and contours of maximum shear strain at the end of analysis (time $t=30 \mathrm{~s}$ ) obtained using 10 sample functions of Type 1 seismic input and 10 sample functions of Type 3 seismic input (shown in Fig. 13). The deformation scale is the same as the geometric scale.

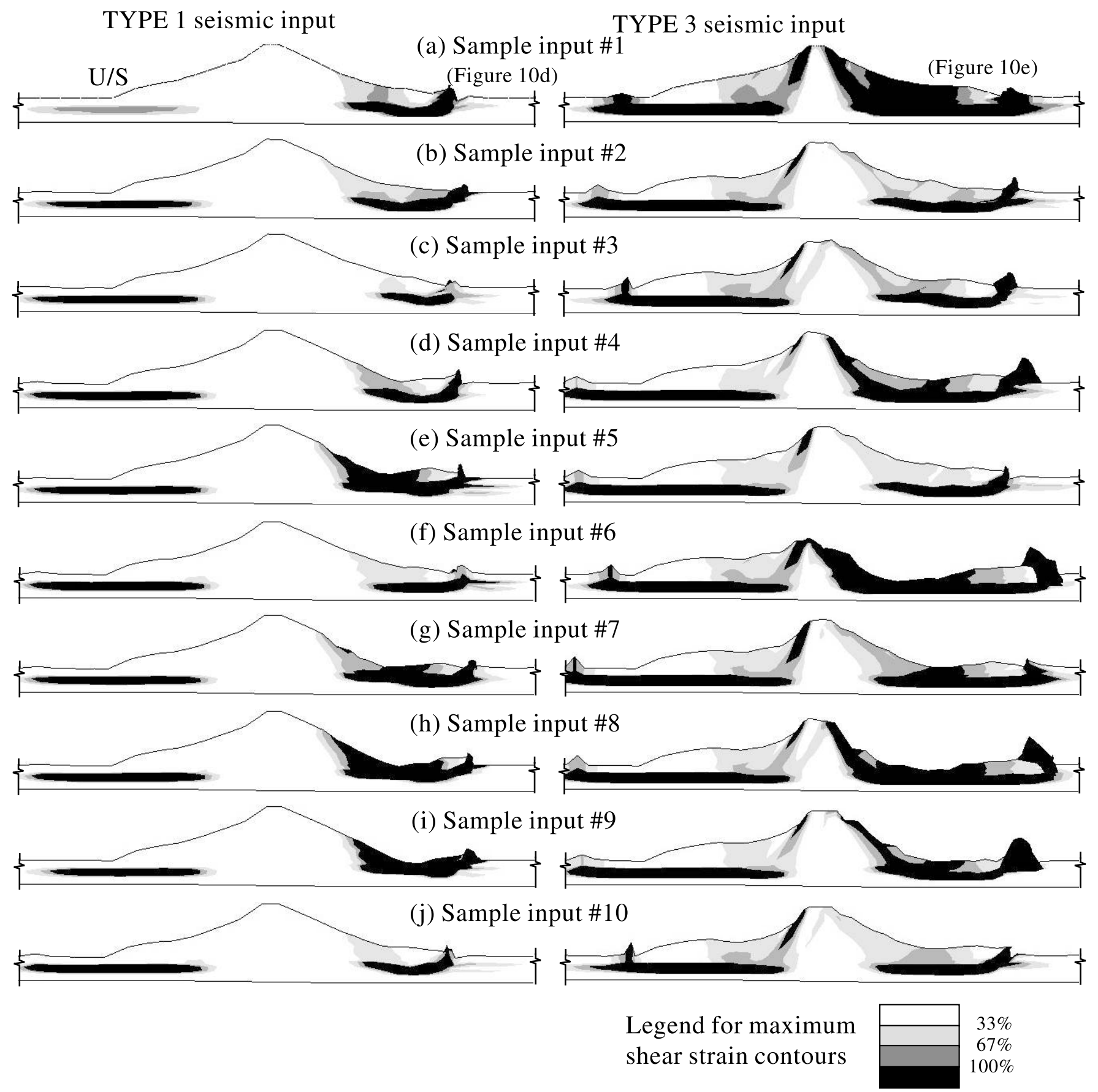

and an embankment dam. A state-of-the-art finite element code that had been repeatedly validated in the past for soil liquefaction analysis was used for this purpose. Some of the numerical results obtained in this study have been verified by comparisons with (1) results of a liquefaction assessment method used in current practice, and (2) centrifuge experimental records.

It was concluded that the interplay between the frequency content of the seismic motion, the vibration characteristics of the structure, and the possible evolution of those characteristics during the shaking had significant influence on the predicted dynamic response. For the soil deposit illustrated in Section 4, significantly larger pore pressures and horizontal displacements at the ground level were predicted for input motions with lower frequency content, especially in the last part of the shaking period when epwp build-up induced more soil softening. For the embankment dam presented in Section 5 and subjected to three different seismic input mo- 
Fig. 15. Predicted crest displacements using 10 sample functions for seismic inputs, compatible with Types 1 and 3 acceleration response spectra.
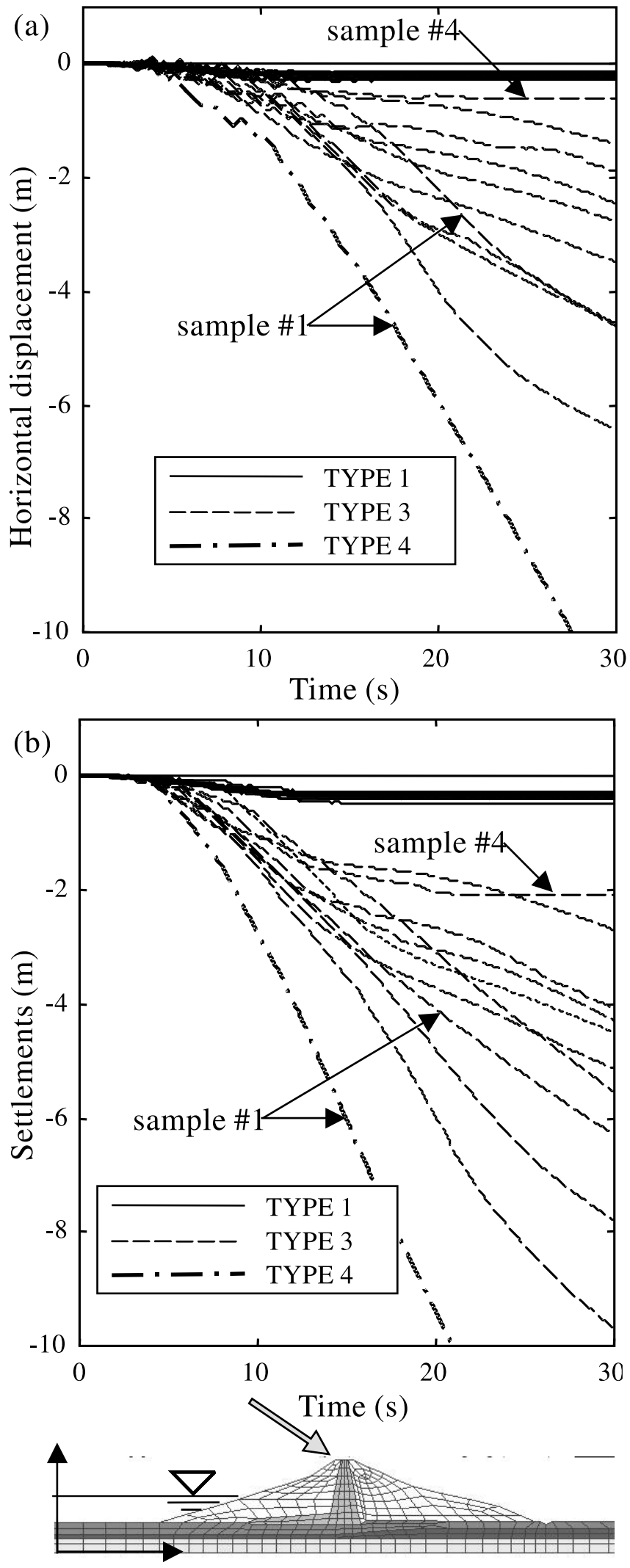

tions, the overall structural response was found to be strongly affected by the frequency content of the input seismic motion. In one case, the numerical model predicted moderate structural damage and deformations that stabilized at the end of shaking. In the other two cases, using seismic input with lower frequency content, the numerical model predicted progressive slope failures after the end of shaking and postearthquake failure of the dam.

Build-up of pore-water pressures and large shear strains may lead to dramatic changes in the vibration characteristics of saturated soil deposits and earth structures during earthquakes. Ground motions having the maximum spectral amplitudes in the range of the new characteristic periods deliver more energy to the system and may produce more damage. Therefore, besides addressing the whole range of possible loading rates at a given location, seismic verification of earth structures has to account for possible changes in the vibration characteristics during dynamic loading. As earth structures, and in particular saturated soils, tend to soften during cyclic loading, they experience a reduction of their characteristic frequency of vibration. Therefore, special attention should be accorded to low frequency seismic inputs.

\section{Acknowledgements}

The work reported here was initiated with sponsorship from the National Science Foundation, under Grant No. BCS-9257900 and was subsequently supported by the Natural Sciences and Engineering Research Council of Canada under Grant No. RG203795-98, and by C-CORE. The author is also indebted to the Department of Civil and Environmental Engineering at Princeton University, and especially to Dr. Jean H. Prevost, for providing the finite element code used in this study.

\section{References}

Abrahamson, N.A. 1993. Spatial variation of multiple support inputs. In Proceedings of the 1st U.S. Seminar on Seismic Evaluation and Retrofit of Steel Bridges, San Fransisco, Calif., Edited by A. Astaneh and J.E. Roberts. University of California, Department of Civil Engineering, Berkeley, Calif.

Arias, A. 1970. A measure of earthquake intensity. In Seismic design for nuclear power plants. Edited by R.J. Hansen. MIT Press. Cambridge, Mass., pp. 438-483.

Biot, M.A. 1962. Mechanics of deformation and acoustic propagation in porous media. Journal of Applied Physics, 33(4):1482-98.

Das, B.M. 1983. Fundamentals of soil dynamics. Elsevier, N.Y.

Deodatis, G. 1996. Non-stationary stochastic vector processes: seismic ground motion applications. Probabilistic Engineering Mechanics, 11: 149-168.

Gulf Canada Resources Inc. 1984. Frontier development - Molikpaq, Tarsiut delineation - 1984/85 season. Technical Report 84F012.

Jennings, P.C., Housner, G.W., and Tsai, N.C. 1968. Simulated earthquake motions. Technical Report, Earthquake Engineering Research Laboratory, California Institute of Technology, Pasadena, Calif.

Juang, C.H., and Jiang, T. 2000. Assessing probabilistic methods for liquefaction potential evaluation. Soil Dynamics and Liquefaction 2000, American Society of Civil Engineers, Geotechnical Special Publication No. 107, pp. 148-162. 
Keane, C.M., and Prevost, J.H. 1989. An analysis of earthquake data observed at the Wildlife Liquefaction Array Site, Imperial County, California. In Proceedings of the 2nd US-Japan Workshop on Liquefaction, Large Ground Deformations, and Their Effects on Lifelines, Grand Island, N.Y., 26-28 Sept. and Itaca, N.Y., 29 Sept. Edited by T.D. O'Rourke and M. Hamada. National Center for Earthquake Engineering Research, Buffalo, N.Y., pp. 39-53.

Madabhushi, S.P.G., and Schofield, A.N. 1993. Centrifuge modelling of tower structures on saturated sands subjected to earthquake perturbations. Géotechnique, 43(4):555-565.

Ohtomo, K., and Shinozuka, M. 1990. Two-dimensional spatial severity of liquefaction. In Proceedings of the 8th Japan Earthquake Engineering Symposium, Tokyo.

Popescu, R. 1995. Stochastic variability of soil properties: Data analysis, Digital simulation, Effects on system behavior. Ph.D. thesis, Princeton University, Princeton, NJ.

Popescu, R., and Prevost, J.H. 1993. Centrifuge validation of a numerical model for dynamic soil liquefaction. Soil Dynamics and Earthquake Engineering, 12(2):73-90.

Popescu, R., and Prevost, J.H. 1995. Comparison between VELACS numerical class 'A' predictions and centrifuge experimental soil test results. Soil Dynamics and Earthquake Engineering, 14(2):79-92.

Popescu, R., Prevost, J.H., Ohbo, N., and Hayashi, K. 1992. Numerical simulations of soil liquefaction, In Proceedings of the 4th US-Japan Workshop on Earthquake Resistant Design of Lifeline Facilities and Countermeasures for Soil Liquifaction, Honolulu, Hawaii, 27-29 May. Edited by T.D. O'Rourke and M. Hamada. National Center for Earthquake Engineering Research, Buffalo, N.Y., pp. 269-282.

Popescu, R., Prevost, J.H., and Deodatis, G. 1997. Effects of spatial variability on soil liquefaction: Some design recommendations. Géotechnique, 47(5):1019-1036.
Popescu, R., Prevost, J.H., and Deodatis, G. 1998. Seismic Analysis of a Wharf Seawall. In Structural Engineering World Wide 1998, Proceedings of the Structural Engineers World Congress (SEWC), 19-23 July. Edited by N.K. Srivastava, G.L. Fenves, R.G. Domer, and A.H.-S. Ang. San Francisco, CA, July.

Popescu, R., Deodatis, G., and Prevost, J.H. 2000. PRISM - a strong motion database. In Proceedings of the 53rd Canadian Geotechnical Conference. Montreal,Que., 15-18 October, Edited by D. LeBoeuf. Canadian Geotechnical Society, Alliston, Ont., Vol. 2, pp. 825-832.

Prevost, J.H. 1985. A simple plasticity theory for frictional cohesionless soils. Soil Dynamics and Earthquake Engineering, 4(1): 9-17.

Prevost, J.H. 1999. DYNAFLOW: a nonlinear transient finite element analysis program. Technical Report, Princeton University, Princeton, NJ.

Seed, H.B., Idriss, I.M., Makdisi, F., and Banerjee, N. 1975. Representation of irregular stress-time histories by equivalent uniform stress series in liquefaction analyses. Technical report, EERC 75-29, University of California, Berkeley.

Seed, R.B., and Harder, L.F. 1990. SPT-based analysis of cyclic pore pressure generation and undrained residual strength. In Proceedings of the H. Bolton Seed Memorial Symposium. Edited by J.M. Duncan. Vol. 2, pp. 351-376.

Steedman, R.S., Ledbetter, R.H., and Hynes, M.E. 2000. The influence of high confining stress on the cyclic behavior of saturated sand. Soil Dynamics and Liquefaction 2000, American Society of Civil Engineers, Geotechnical Special Publication No. 107, pp. $35-57$.

Uniform Building Code. 1994. International Conference of Building Officials, ICBO, Whittier, CA. Vol. 2. 